\title{
Numerical Evaluation of the Effect of Gradient on Reflection Coefficient of Continuously Graded Layer
}

\author{
Ahmed Markou, Hassan Nounah \\ Laboratory of Metrology and Information Processing, Department of Physics \\ Faculty of Sciences, Ibnou Zohr University \\ Agadir, Morocco
}

\begin{abstract}
This paper presents a numerical model, based on transfer matrix method, for modeling the propagation of surface acoustic waves at the interface formed by the coupling liquid and a continuously inhomogeneous thin layer on a semi-infinite substrate. The tow-dimensional spectrum of reflection coefficient computed by this model, allows determining the modes which propagate at the studied interface, this model treats different profile of gradients, and the numerical results obtained show that the reflection coefficient is sensitive to the variation of these gradients.
\end{abstract}

Keywords-surface acoustic waves; nondestructive testing; functionally graded materials; dispersion curve; Lamb modes

\section{INTRODUCTION}

The functionally graded materials are recently developed in Japan for to be used as thermal barrier materials for aerospace structures and fusion reactors (high temperature applications), and now they are developed for the general use in different engineering applications. The FGMs are made from different of material constituents, where their properties such as elastic constants and density are varying according to the spatial coordinates [1]. The profile of gradient in physical properties obtained is governed by the procedure of fabrications [2][3]. For modeling FGMs, tow approaches are possible. The first assumed that the gradient is piecewise varying, and the FGM is slicing into finite homogeneous layers [4][5]. The second approach assumed that the gradient is varying continuously between the tow basic material properties [6]. Due to their complex structure, the characterization of FGMs poses a great challenge. The surface acoustic wave is widely used for characterizing the profile of gradient near to their surface [7] because surface acoustic wave (SAW) propagation, is strongly dependent to the local properties of the materials [8]. The good Knowledge of the reflection coefficient is necessary to determine and analyze the reflected or transmitted modes propagating at the interface of studied structures [9].

In inhomogeneous medium, the equation governing the propagation of elastic wave, is a system of three second order differential equations with no constant coefficients, for the displacement field, in which involved spatial derivatives of the elasticity coefficients, these type of equations can be solved analytically for specific profile of gradients [10][11][12].

In the present article, we have used the transfer matrix method [13][14] and the theory developed by L.M.Brekhovskikh [15] to compute the reflection coefficient and the dispersion curves of different numerical profiles of gradient simulating an FGM structure. The numerical sample considers the transversal cut near to the surface of titanium plate after the oxidation process, where the titanium layer presents a continuous gradient in its elastic properties crossing its depth. The inhomogeneous area is divided into some elementary layers with the same thickness. The number of elementary layers is selected such that the error rate on the velocity of the Rayleigh mode is less than one percent in the frequency range.

\section{THEORY AND METHOD}

The geometry of the problem is illustrated in the Fig.1. At each layer the elastic properties are constants, and then, the continuous gradient is replaced by piecewise constant functions. The minimal slicing to ensure reliable results and easy calculations are determined. (Fig.2)

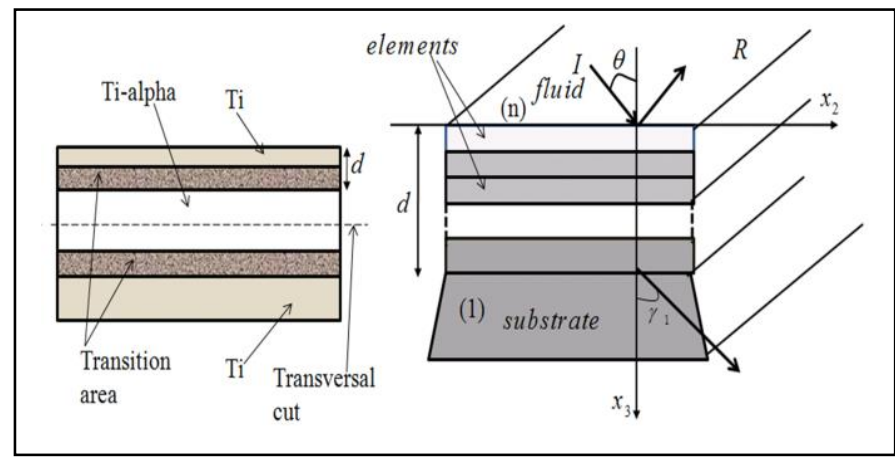

Fig. 1. Geometry of graded layer on sem-infinte substrate

The gradient variations are in the direction $\left(\mathrm{ox}_{3}\right)$, and then, all properties of the material are only depending to the $x_{3}$ coordinate. Taken account of the boundary conditions, the displacement -stress vector at each tow adjacent layers can be written as following [15]:

$$
\xi^{m}\left(x_{3}\right)=A^{m}\left(x_{3}\right) \xi^{m-1}\left(x_{3}\right)
$$

Where $\xi$ and $A$ are, respectively, the displacement-stress vector and transfer matrix of the layer $m$, such as:

$$
\xi^{m}=\left[\begin{array}{llll}
u_{11}^{m} & u_{33}^{m} & \sigma_{33}^{m} & \sigma_{13}^{m}
\end{array}\right] \text { And } A^{m}=a_{+}^{m}\left(a_{-}^{m}\right)^{-1} \text {, these }
$$

tow matrices are obtained for the same layer $m$ (at its limits, upper and lower interface), they contain the same properties of 
the layer $m$, but they differ by the term which contains the expression $\exp \left(j k x_{3}\right)$ [15].

In the general case, the displacement-stress vector has six components and the matrix $A$ is at sixth order [16]. But according to the theory developed by L.M.Brekhovskikh, the calculation of the reflection coefficient requires only fourth components of displacement-stress vector [15].

Step by step, using (1), and taken account the boundary conditions at the interface separating tow successive layers, it is possible to express the displacement-stress vector at the interface liquid/first elementary layer $\left(\mathrm{x}_{3}=0\right)$ as a function of that at the interface last elementary layer/ substrate $\left(\mathrm{x}_{3}=\mathrm{d}\right)$ :

$$
\xi^{n}=A^{n} A^{n-1} \ldots A^{1} \xi^{1}
$$

And then, the transfer matrix of the continuous graded layer is as following ( $n$ is the number of elementary layers):

$$
A=\prod_{n}^{1} A^{m}
$$

At the interface liquid/layer the shear component $\sigma_{13}^{n}$ is null (the liquid is considered perfect, its shear modulus is then null), and then from (2) and (3), we can deduced the following equation:

$$
A_{41} u_{11}^{1}+A_{42} u_{33}^{1}+A_{43} \sigma_{33}^{1}+A_{44} \sigma_{13}^{1}=\sigma_{13}^{n}=0
$$

By using (2) and (4), we deduce the following system of equations:

$$
\begin{aligned}
& u_{33}^{n}=M_{22} u_{33}^{1}+M_{23} \sigma_{33}^{1}+M_{24} \sigma_{13}^{1} \\
& \sigma_{33}^{n}=M_{32} u_{33}^{1}+M_{33} \sigma_{33}^{1}+M_{34} \sigma_{13}^{1}
\end{aligned}
$$

With:

$$
M_{p i}=A_{p i}-\frac{A_{4 i}-A_{p 1}}{A_{41}}, p=2,3 \text { and } i=2,3,4 .
$$

At the tow interfaces liquid/first elementary layer and last elementary layer/substrate, the normal displacement and stress components can be written as following [14]:

$$
\begin{aligned}
& u_{33}^{n}=j \alpha_{n} \phi_{i n c}(1-R) \\
& \sigma_{33}^{n}=-\omega^{2} \rho_{n} \phi_{i n c}(1+R)
\end{aligned}
$$

$\Phi_{i n c}$ and $R$ (quotient between amplitudes of incident and reflected waves) and $\rho_{n}$ and $\alpha_{n}$ are respectively, the amplitude of incident acoustic wave and reflection coefficient and density and wave number in coupling liquid. $\omega$ is the angular frequency of acoustic wave. $\theta$ is the incident angle of acoustic wave. $\theta_{1}$ and $\gamma_{1}$ are the refraction angles of transmitted waves in the substrate. $d$ is the thickness of graded layer.

$$
\begin{aligned}
& u_{11}^{1}=j\left(\mathrm{~W} k_{L}^{1} \sin \theta+P k_{T}^{1} \cos \gamma_{1}\right) \\
& u_{33}^{1}=j\left(-W k_{L}^{1} \cos \theta_{1}+P k_{L}^{1} \sin \theta\right) \\
& \sigma_{33}^{1}=-\omega^{2} \rho_{1}\left(W \cos 2 \gamma_{1}-P \sin 2 \gamma_{1}\right) \\
& \sigma_{13}^{1}=\omega^{2} \rho_{1}\left(P \cos 2 \gamma_{1}+W \frac{C_{T 1}^{2}}{C_{L 1}^{2}} \sin 2 \theta_{1}\right)
\end{aligned}
$$

$C_{L 1}$ and $C_{T 1}$ are the velocities of longitudinal and shear waves in the substrate. $\mathrm{P}$ and $\mathrm{W}$ (amplitudes of acoustic waves in the substrate) are tow unknowns, and only the rate $\frac{P}{W}$ is necessary to determine the reflection coefficient. This quotient can be deduced from (4) [15].

Finally, $R$ is the reflection coefficient, it is a function of incident angle and frequency. By using (4) and (5) and (6) and (7), its expression can be written in the following form:

$$
R(\omega, \theta)=\frac{\frac{\sigma_{33}^{n}}{\omega u_{.33}^{n}}-j \omega \frac{\rho_{n}}{\alpha_{n}}}{\frac{\sigma_{33}^{n}}{\omega u_{33}^{n}}+j \omega \frac{\rho_{n}}{\alpha_{n}}}
$$

The tangent hyperbolic profile of longitudinal and transversal velocities is given by the following formula:

$$
C=C_{0}+\left(C_{d}-C_{0}\right)\left[\frac{\tanh \left(a\left(\mathrm{x}_{3}-\frac{d}{2}\right)\right)-\tanh \left(-a\left(\frac{d}{2}\right)\right)}{\tanh \left(a \frac{d}{2}\right)-\tanh \left(-a \frac{d}{2}\right)}\right] \text { (9) }
$$

$\mathrm{C}_{0}$ and $\mathrm{C}_{\mathrm{d}}$ are the ultrasonic velocities in the surface of layer and in the substrate. $a$ is a given parameter.

\section{NUMERICAL RESULTS AND DISCUSSION}

The ultrasonic velocities in substrate of Titanium alphacase and in Titanium layer [18] and the numerical data used in simulations are regrouped in the table I. The coupling liquid surmounted this structure is the water.

\section{TABLE I. INPUT DATA USED IN SimULATIONS}

\begin{tabular}{|l|l|l|l|l|}
\cline { 2 - 5 } \multicolumn{1}{c|}{} & $\mathbf{V}_{\mathbf{L}}(\mathbf{m} / \mathbf{s})$ & $\mathbf{V}_{\mathbf{T}}(\mathbf{m} / \mathbf{s})$ & $\boldsymbol{\rho}\left(\mathbf{k g} / \mathbf{m}^{\mathbf{3}}\right)$ & Thickness $(\boldsymbol{\mu m})$ \\
\hline $\begin{array}{l}\text { Titanium } \\
\text { alpha-c }\end{array}$ & 6660 & 3553 & 4460 & - \\
\hline Titanium & 6060 & 3230 & 4460 & 100 \\
\hline Water & 1500 & - & 1000 & - \\
\hline
\end{tabular}

\section{A. Stabilization of the velocity of Rayleigh mode}

To determine the number of elementary layers sufficient to ensure the accuracy of calculations, the frequency is fixed at $150 \mathrm{MHz}$ and the input number of elementary layers is increasing until the stabilization of velocity of Rayleigh mode. 
In the Titanium, the wavelength of Rayleigh at the frequency of $150 \mathrm{MHz}$ is $\lambda_{R}=20 \mu \mathrm{m}$, which is less than thickness of the graded layer $(d=100 \mu m)$. About twelve layers the velocity of Rayleigh mode (for linear profile) becomes constant and stabilizes at the $3020.30 \mathrm{~m} / \mathrm{s}$ and the relative error is null (Fig. 2). For example, at teen layers the velocity of Rayleigh mode is $3010.10 \mathrm{~m} / \mathrm{s}$ and the error is about $0.34 \%$. For a graded aluminum layer on the substrate of silica, for to have an error less than $1 \%$, it should about twenty layers [4][5][17]. This difference can be explained by the significant difference between the properties of the graded layer and those of substrate, in contrast to the Titanium alpha and Titanium where their properties are very close and the step of variation of the profile is weak, then, the continuity is assured only for several elementary layers.

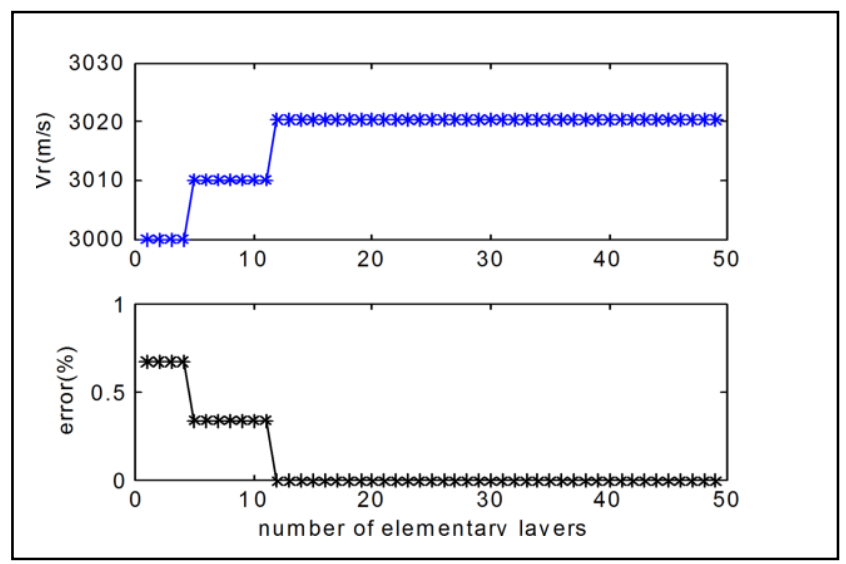

Fig. 2. Variation of the velocity of Rayleigh mode with elementary layers

\section{B. Reflection coefficient and dispersion curve}

The reflection coefficient in (8) is computed for each frequency at each incident angle. The length of vectors which contain frequencies and incident angles are respectively choosing 401 and 451 elements. The step between tow successive frequencies is fixed at $\Delta f=0.375 \mathrm{MHz}$ in the frequency range of $[0150 \mathrm{MHz}$. For the incident angles the step is $\Delta \theta=0.1089$ degree for the incident angle range of [0 50 degree]. The reflection coefficient is then a matrix of $401 \times 451$ elements. We have remarked that the image of the phase of this matrix (or its imaginary or its real part) gives the dispersion of the generalized Lamb modes reflected by the studied structure (fig. 3 and Fig.4).

We have remarked that the counter of the image in Fig. 3 can clearly show all generalized modes reflected in the coupling liquid (Fig.4), this method gives the dispersion curve from reflection coefficient without solving (4).

The image in Fig.3 shows a series of modes which propagate at the interface of studied structure. Tow types of acoustic surface modes are present:

\section{The Rayleigh and pre-Rayleigh modes (sezawa modes):}

The first mode is the Rayleigh mode (Rayleigh wave), its velocity decrease, with frequency, from $4020 \mathrm{~m} / \mathrm{s}$ to reach the asymptotic value of $3020.30 \mathrm{~m} / \mathrm{s}$ at high frequencies. The velocity of first Sezawa mode decrease from the $6612 \mathrm{~m} / \mathrm{s}$ (the longitudinal velocity in substrate is $6660 \mathrm{~m} / \mathrm{s}$ ) to the asymptotic value of $3342 \mathrm{~m} / \mathrm{s}$ (the transversal velocity in layer is $3230 \mathrm{~m} / \mathrm{s}$ ). Other modes (sezawa modes) vary between 5600 $\mathrm{m} / \mathrm{s}$ and the asymptotic velocity of $3342 \mathrm{~m} / \mathrm{s}$ at high frequencies.

Higher order modes: which decrease rapidly from high velocities to the velocity of longitudinal mode in the layer (the asymptotic value of $6100 \mathrm{~m} / \mathrm{s}$ ) (Fig.4).

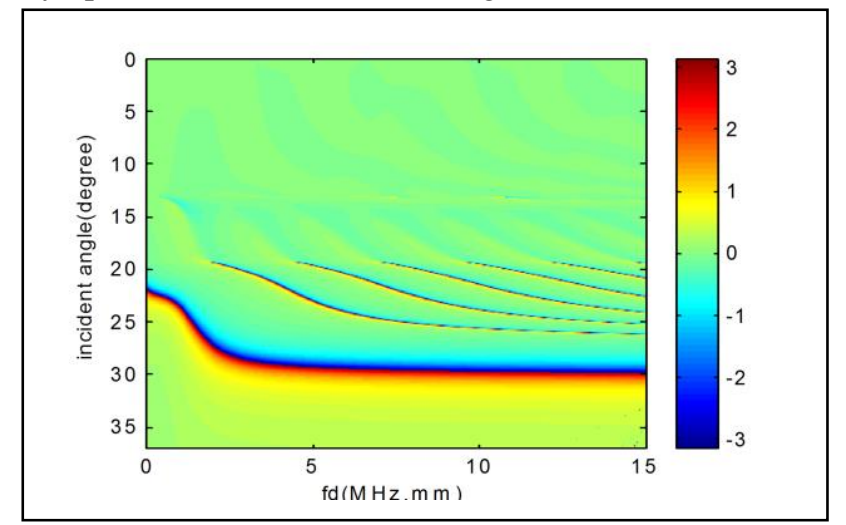

Fig. 3. Dispersion image for linear profile-phase of the matrix $\mathrm{R}$

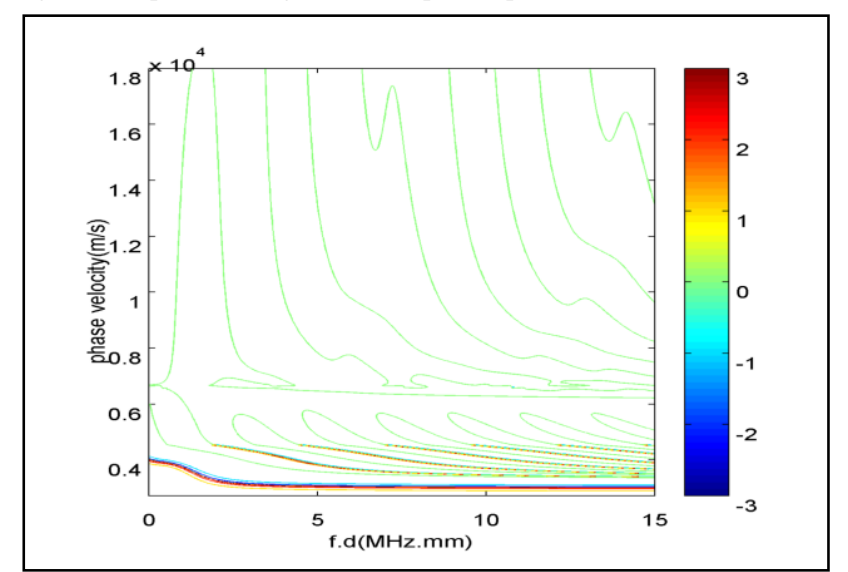

Fig. 4. Contour of the image in Fig. 3

At low frequencies $(f=0.375 \mathrm{MHz}$, Fig.5 $)$ and at the incident angle of $\theta=22$ degree, only the Rayleigh mode is existing, its acoustic energy is totally reflected by the interface liquid/graded layer $(R=1)$. When the frequency increases, many modes appear at different incident angles (Fig.6). Their velocities can be determined by using the following formula:

$$
v=\frac{v_{l i q}}{\sin \theta_{c}}
$$

$v_{\text {liq }}$ is the longitudinal velocity in the coupling liquid and $\theta_{\mathrm{c}}$ is the critical incident angle. This angle can be determined from the phase of the reflection coefficient, it corresponds to the pick figured in the phase of the reflection coefficient (Fig.5).

The phase velocity of the Rayleigh mode and Sezawa mode is determined by using (10). At each frequency, the critical angles are determined by using an appropriate algorithm. The dispersion curve of these tow modes is presented in Fig.7. 

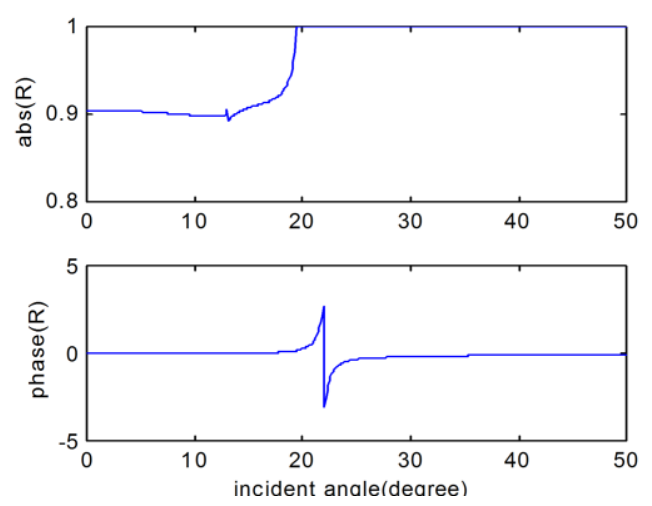

Fig. 5. Modulus and phase of the $R C$ at $f=0.375 \mathrm{MHz}$
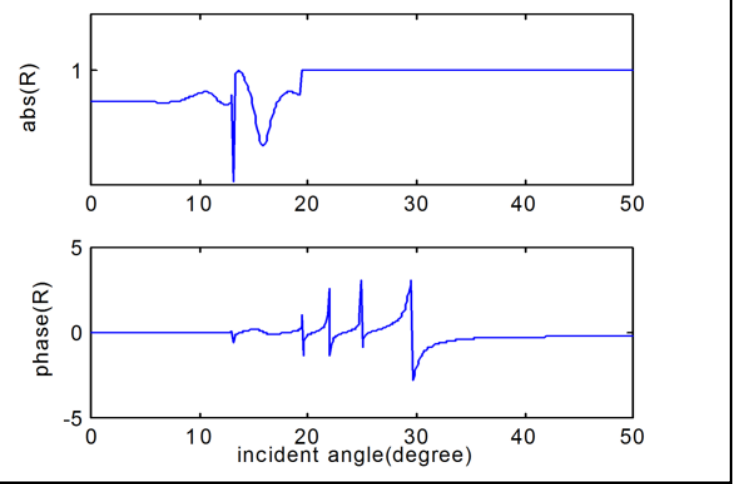

Fig. 6. Modulus and phase of the $R C$ at $f=74.62 \mathrm{MHz}$

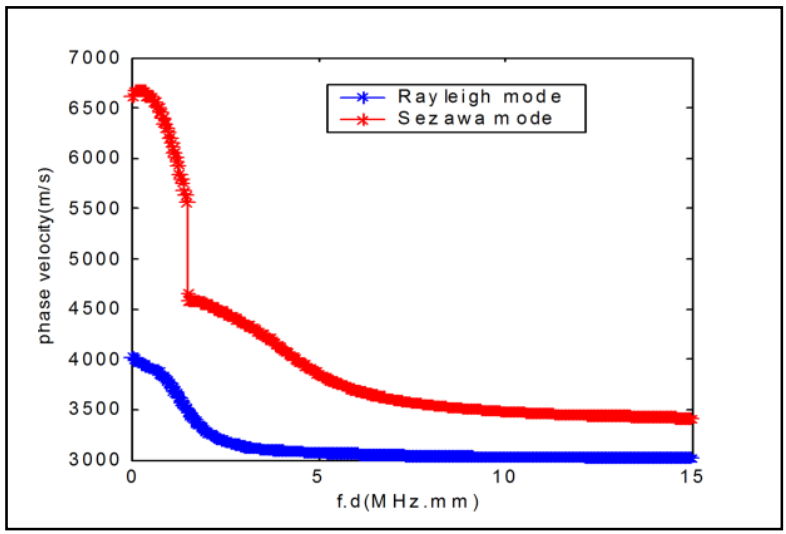

Fig. 7. Dispersion curve of Rayleigh and sezawa modes

\section{C. effect of the gradient on reflection coefficient}

In the case of the inverse problem i.e. when the reflection coefficient and dispersion curve are known and elastic properties of the studied structure are unknown, in this case, the complete characterization of continuously graded profile is very delicate.

However, the reflection coefficient is the good indicator of the profile of heterogeneity of graded materials.

The modulus of reflection coefficient for different profiles is presented in the following figures:

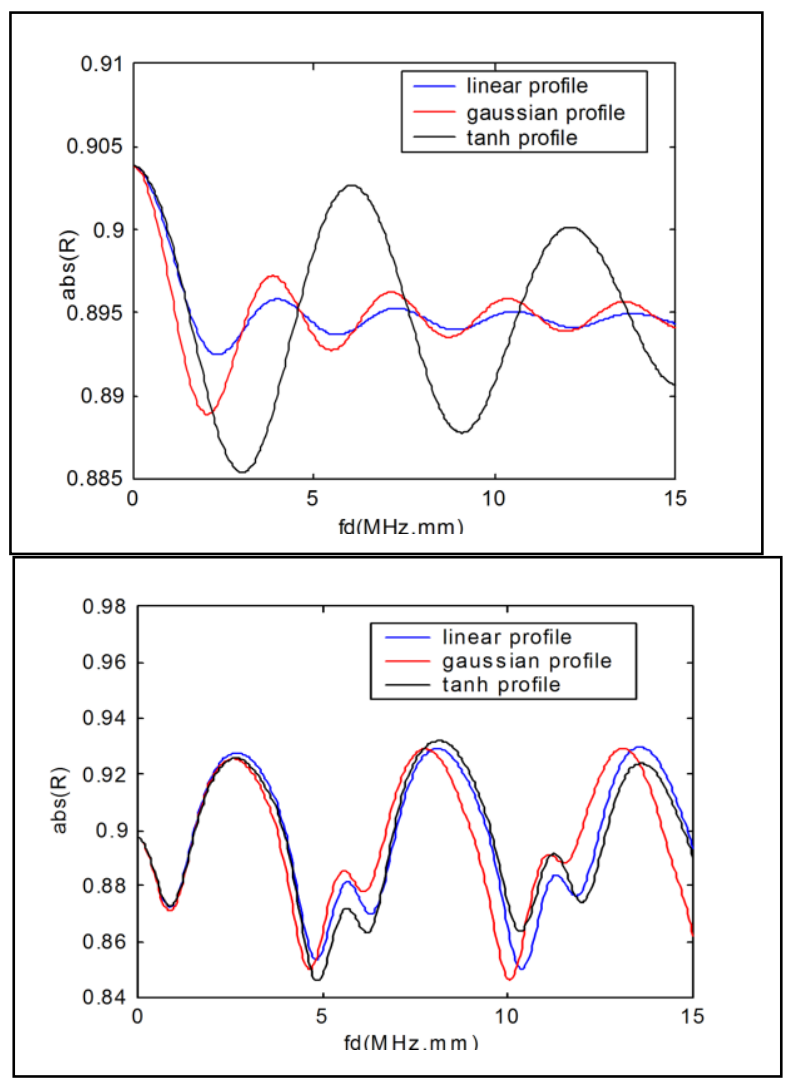

Fig. 9. Modulus of the reflection coefficient at $\theta=11$ degree

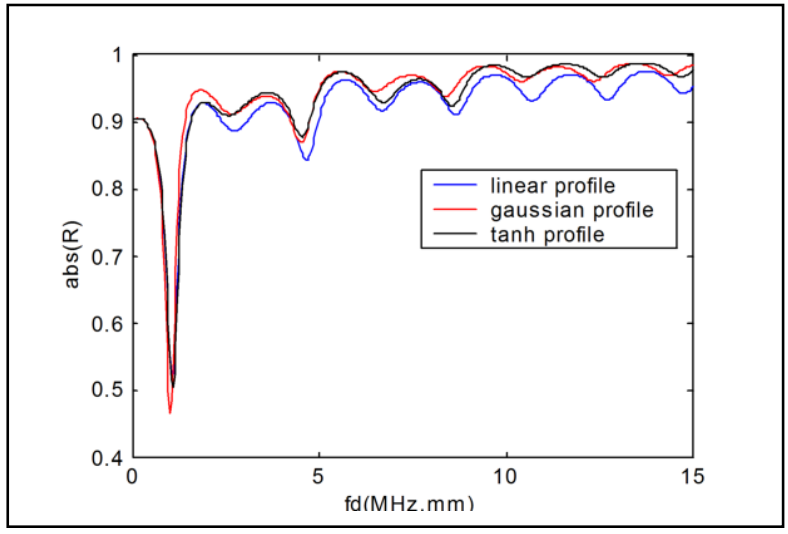

Fig. 10. Modulus of the reflection coefficient at $\theta=14$ degree

From the Fig.8 and Fig.9 and Fig.10, we can observe the influence of the profile of heterogeneity on the spectrum of reflection coefficient and we note the following remarks:

- The resonance in the reflection coefficient.

- The reflection coefficient is sensitive to the shape of gradient at certain frequency range and incident angles.

- At normal incidence, the reflection coefficient is more sensitive to the profile of gradient (Fig.8).

- At low frequencies (0 to $20 \mathrm{MHz}$ ), the reflection coefficient is insensitive to the nature of the gradient. 


\section{Interpretations:}

- At normal incidence, the acoustic wave propagates in the direction of the gradient, the vertical interference with the heterogeneity occurs.

- At low frequencies, the wavelength is important compared to the thickness of graded area, the whole of acoustic energy is located in the substrate.

- At high frequencies, the wavelength is weak compared to the thickness of the heterogeneous area, the acoustic wave is sensitive to the spatial variation of gradient.

\section{Study of Rayleigh and Sezawa modes}

For exciting only these tow first modes, the incident angle is fixed at the critical angle of $\theta=26$ degree, which correspond to the velocity of $3421 \mathrm{~m} / \mathrm{s}$ for the both modes (Fig.11).

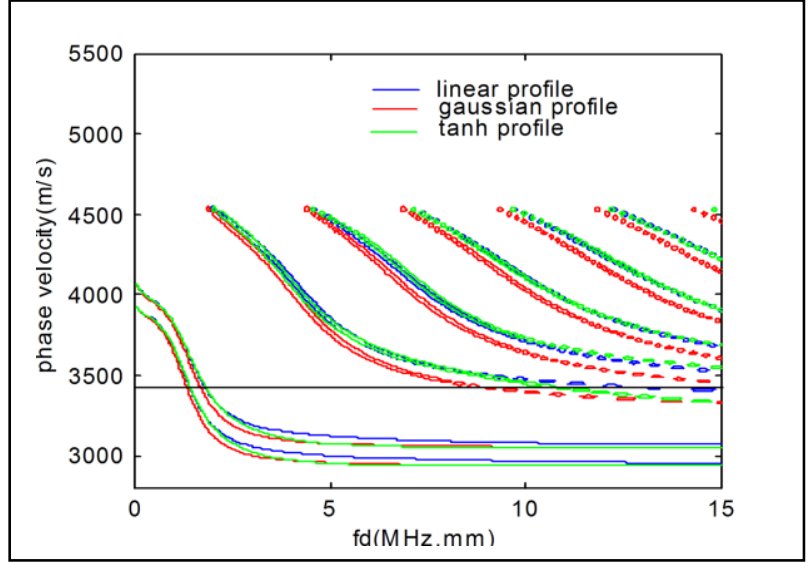

Fig. 11. Dispersion curve for Rayleigh and Sezawa modes-real part of the $R$

At this incident angle, the modulus of reflection coefficient is equal to one in all frequency range (Fig.5 and Fig.6). For evaluating the influence of the profile of the gradient, the phase of reflection coefficient gives the best results.

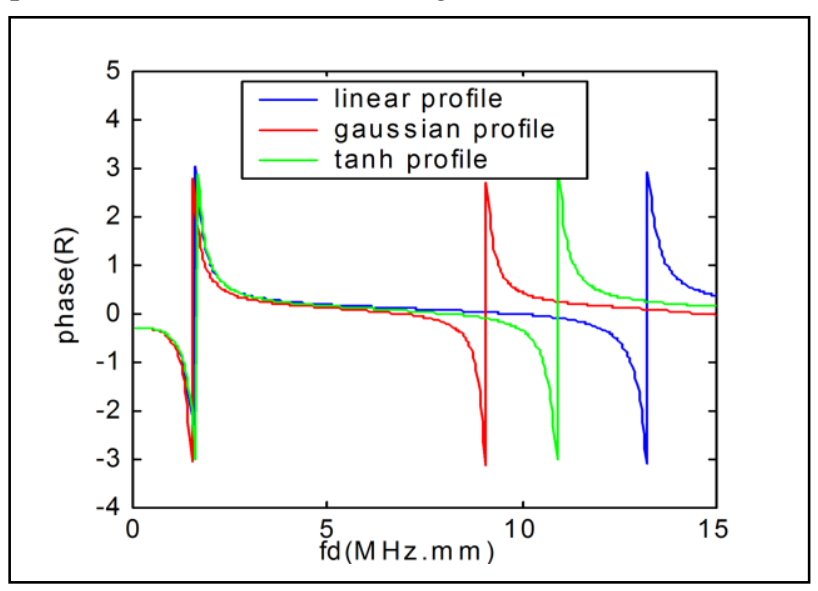

Fig. 12. Phase of the $\mathrm{RC}$ at $\theta=26$ degree for Rayleigh and sezawa modes

From the Fig.12, we can observe the influence of the nature of the gradient on the phase of reflection coefficient for the Sezawa mode. The frequency of existence of this mode depends to the shape of gradients. The Rayleigh mode dose not influenced by the profile of gradient (fig.12). The results are regrouping in table II:

TABLE II. FREQUENCY OF EXCITATION OF THE TOW FIRST MODES

\begin{tabular}{|c|c|c|c|}
\hline & gaussian pr. & linear pr. & $\tanh$ pr. \\
\hline \multicolumn{4}{|c|}{ Rayleigh mode } \\
\hline $\mathrm{f}(\mathrm{MHz})$ & 15.375 & 16.125 & 16.50 \\
\hline$\lambda(\mu \mathrm{m})$ & 222.55 & 212.20 & 207.38 \\
\hline \multicolumn{4}{|c|}{ Sezawa mode } \\
\hline $\mathrm{f}(\mathrm{MHz})$ & 91.125 & 132.75 & 109.5 \\
\hline$\lambda(\mu \mathrm{m})$ & 37.550 & 27.776 & 31.249 \\
\hline
\end{tabular}

The second mode (or sezawa mode) is excited at high frequency $(\lambda<d)$ that's why the phase of reflection coefficient of this mode is more sensitive to the profile of gradients (table II).

At the incident angle of $\theta=26$ degree, the wavelength of the Rayleigh mode is important than thickness of graded layer, the acoustic wave is not affected by the heterogeneous area $(\lambda>d)$ (Fig12), (table.II).

When the incident angle increases, the Rayleigh mode (first mode) is excited at high frequencies and becomes sensitive to the profiles $(\lambda<d)$ (Fig.13) (Table.III ).

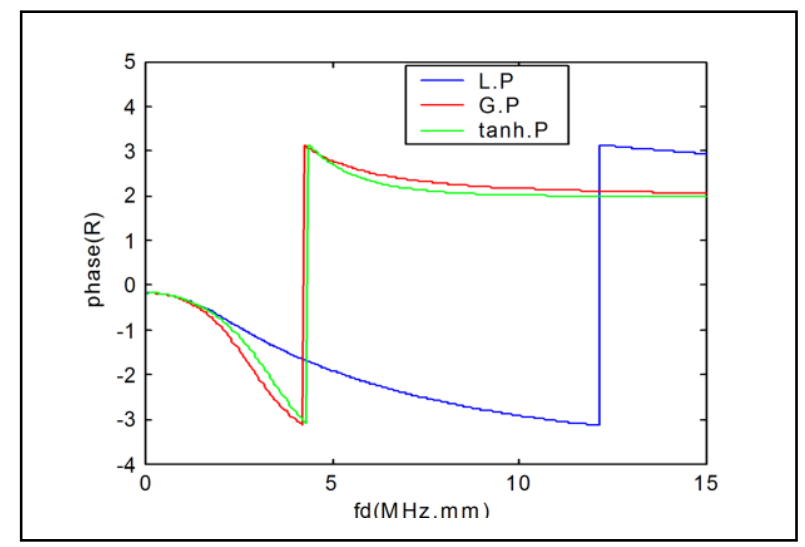

Fig. 13. Phase of the $\mathrm{RC}$ for Rayleigh mode at $\theta=30$ degree

TABLE III. FREQUENCY OF ECXITATION OF RAYLEIGH MODE

\begin{tabular}{|c|c|c|c|}
\hline & gaussian pr. & linear pr. & tanh. pr. \\
\hline \multicolumn{4}{|c|}{ Rayleigh mode } \\
\hline $\mathrm{f}(\mathrm{MHz})$ & 49.875 & 121.500 & 48.375 \\
\hline$\lambda(\mu \mathrm{m})$ & 60.557 & 24.858 & 62.435 \\
\hline
\end{tabular}

\section{CONCLUSION}

This numerical study, based on transfer matrix method, of the propagation of acoustic wave in continuously graded thin layer on semi-infinite substrate, aims to understand the behavior of acoustic wave at the interface of such structure, in order to discover an efficient tool for characterizing the gradient in elastic properties present near to the surface of these structures. 
We used the model described in this article to compute the tow-dimensional spectrum of the reflection coefficient which is a good indicator of the heterogeneity of materials. At normal incidence and at high frequencies, the reflection coefficient is more sensitive to the gradient. For the Rayleigh and Sezawa modes, the phase of the reflection coefficient is best for characterizing the profile of heterogeneity at high frequencies. The frequency of excitation of these modes is influenced by the shape of gradient.

The gradient has little influence on the velocity of Rayleigh and Sezawa modes and this due to the nature of propagation of these modes, which propagate perpendicularly to direction of the heterogeneity, the vertical decreasing of the displacement field is very rapid, and then, the vertical interferences are very weak.

The choice of the incident angle and the frequency is mandatory for ensuring best characterization of FGMs.

\section{REFERENCES}

[1] Suresh, S., M. A., " Fundamentals of functionally graded materials", 1998.

[2] S.Y. Sung, Y.J. Kim, “ Alpha-case formation mechanism on titanium investment castings”, Materials Science and Engineering A 405 (2005) $173-177$.

[3] J. F. Groves and H. N. G. Wadley, "Functionally graded materials synthesis via low vacuum directed vapor deposition", Composites Part B 28B(1997), 57-69.

[4] H.Nounah, B.Cros, and J.Attal, "Theoretical approch of the characterization of gradients in elastic properties by acoustic microscopy", Eur. Phys. J. AP 5, 221-226, 1999.

[5] H. Nounah, B. Cros and J. Attal,"Modelling of gradients in elastic properties for acoustic microscopy characterizations", Eur. Phys. J. AP 6 (3) 237 (1999).
[6] Shuvalov A. L., Le Clezio E., Feuillard G., "The state-vector formalism and the Peano-series solution for modelling guided waves in functionally graded anisotropic piezoelectric plates", Int. J. Eng. Sci., 46, 929-47, 2008.

[7] A.Atalar, H.Koymen and L.Degertekin, "Characterization of layred materials by the lamb wave lens", proc. of ultrasonic symposium, New York, 1989.

[8] J. Kushibiki, T. Ishikawa, and N.Chubachi, "Cut-off characterestic of leaky sezawa and pseudo-sezawa wave modes for thin-film characterization”, Appl. Lett.57(19), 5 November 1990.

[9] D.L.Folds, C.D.Loggins, "Transmission and reflection of ultrasonic waves in layered media ", J.Acoust.Soc.Am., 62, 1102-1109, 1977.

[10] Liu, J. Y., Tsai, S. H., Wang, C. C., and Chu, C. R., "Acoustic wave reflection from a rough seabed with a continuously varying sediment layer over-lying an elastic basement",Journal of Sound and Vibration 275, 3-5 (2004),739-755.

[11] Chiu, T.-C., and Erdogan, F., "One-dimensional wave propagation in a functionally graded elastic medium", Journal of Sound and Vibration 222, 3(1990), 453-487.

[12] Robins, A. J. "Reflection of plane acoustic-waves from a layer of varying density", Journal of the Acoustical Society of America 87, 4 (1990), 1546-1552.

[13] W.T.Thomson, "Transmission of elastic waves through a stratified solid medium ", J.Appl.phys., 21, 89-93 , 1950.

[14] N.A. Haskell, "The dispersion of surface waves on multilayered media" , Bull. Seismol. Soc. Am. 43, 377-393, 1953.

[15] L.M. Brekhovskikh, "Waves in layered media ", academic press, New York, 1960.

[16] Adnan H. Nayfeh, Wael G. Abdelrahman, and Peter B. Nagyc, "Analyses of axisymmetric waves in layered piezoelectric rods and their composites", J. Acoust. Soc. Am. 108 (4), October 2000.

[17] H. Nounah, "Modélisation et caractérisation des matériaux à gradient de propriétés mécaniques par des méthodes microacoustiques", phd thesis, Montpellier II, France, 1995.

[18] C. Baron, " le developemen en serie de peano du matricant pour l'étude de la propagation des ondes élastiques en milieux à propriétés continûment variables", Phd thesis, France, 2005. 